\title{
Investigation and Analysis of Medical Device Contamination; Practical Applications of Light and Electron Microscopy
}

\author{
Brown, Richard S. ${ }^{1} \mathrm{MS}$. \\ ${ }^{\mathrm{L}}$ MVA Scientific Consultants, Inc., Duluth, GA 30096, United States.
}

A medical device is essentially anything that makes contact with a patient directly or produces emissions that does not react chemically or has to be metabolized to produce a desired effect[1]. The investigation of medical device product complaints, contamination, discoloration and/or performance involves the documentation and sampling of contact traces using small particle handling techniques, microextraction techniques and the ability to perform multiple analyses on single, small particles or aggregates of materials using a variety of microscopic techniques. The microscopic techniques used by the investigator/microscopist include, but are not limited to polarized light microscopy (PLM), Fourier transform infrared microspectroscopy (FTIR), scanning electron microscopy-energy dispersive x-ray spectrometry (SEM-EDS), confocal Raman microscopy (CRM) and analytical electron microscopy (AEM).

This type of investigation involving contact traces is unique. There is not a standard operating procedure for the identification of all "unknown particles" The investigator/microscopist has a working knowledge of the capabilities and limitations of the microscopic techniques employed and should provide his customer or project leader with a picture of how the analysis will proceed depending on what types of materials are discovered. In this type of an investigation, a single procedure or a single analysis scheme is not applicable as the types of analyses depend entirely on the types of materials discovered during the investigation. Many times the microscopist is providing images of particles adhering to, encapsulated by and reacting with surfaces of the device in ways that have not been seen or recorded previously. The investigative procedure that has been developed over time enables a trained microscopist to rapidly characterize contaminants and produce an analysis scheme to provide a maximum amount of information from a variety of microscopic tests with a minimum amount time. The practical application of different microscopical tests has been reported previously[2].

Microscopy is especially suited for this type of investigation. A skilled microscopist can sample contaminated areas on a medical device and characterize the contaminants using a combination PLM, FTIR and SEM-EDS. CRM and AEM are also useful in the analysis of smaller particles, thin films and nanoparticles. In general, particles removed from a device that are representative of the contamination are initially placed on a beryllium planchet for analysis by SEM-EDS. Typically, this first pass with the SEM-EDS is non-destructive and the same sample can be further analyzed by PLM and FTIR. Opaque particles, polymer particles with fillers and metals may be best addressed by SEM-EDS. SEM-EDS is useful for determining the elemental composition of contamination/debris when drugs or radio opaque materials are suspected to have reacted with a device or delivery tube causing a blockage or damage that resulted in delamination, swelling or discoloration. Low vacuum (LV) SEM-EDS is especially useful when a small device cannot be sectioned for analysis, or when the entire device or section must be examined directly without coating with a conductive film. To avoid misinterpretation of the elemental data produced due to the beam "skirt", EDS spectra must be collected from multiple areas on the sample to account for element contributions due to the larger probe size at higher pressures[3]. PLM can be used to examine residues to determine if they are materials foreign to the device such as mold, 
fungus, minerals, metal, polymer, elastomeric, blood, food, medicine, hair, crystalline, non-crystalline or an aggregate of different materials. Polymeric materials are probed and pressed with a cover slip to observe the behavior of the particles under compression; (plastic or elastomeric), then analyzed by FTIR to characterize the material. Hard particles are crushed and provide multiple fractured pieces to examine by PLM and SEM-EDS. Micro-extractions are used to isolate water soluble and solvent soluble materials that can be further characterized by FTIR[4].

Communication between the investigator and the customer/project manager must be maintained to determine if the device can be cut, sectioned, disassembled, or altered to perform the analysis required. If the device cannot be sectioned or otherwise altered, this information must be conveyed to the investigator before any analysis is performed. The initial investigator must not consume the entire sample during his analysis. A sample of the materials/contamination should be preserved to allow any additional examinations that may be requested including any new techniques that may be developed in the future. Since the process is investigative, the results should be considered preliminary until a final report is prepared. Contact with the customer/project manager is essential to develop investigative leads and not jump to erroneous or spontaneous conclusions that may hinder the determination of the root cause of the contamination.

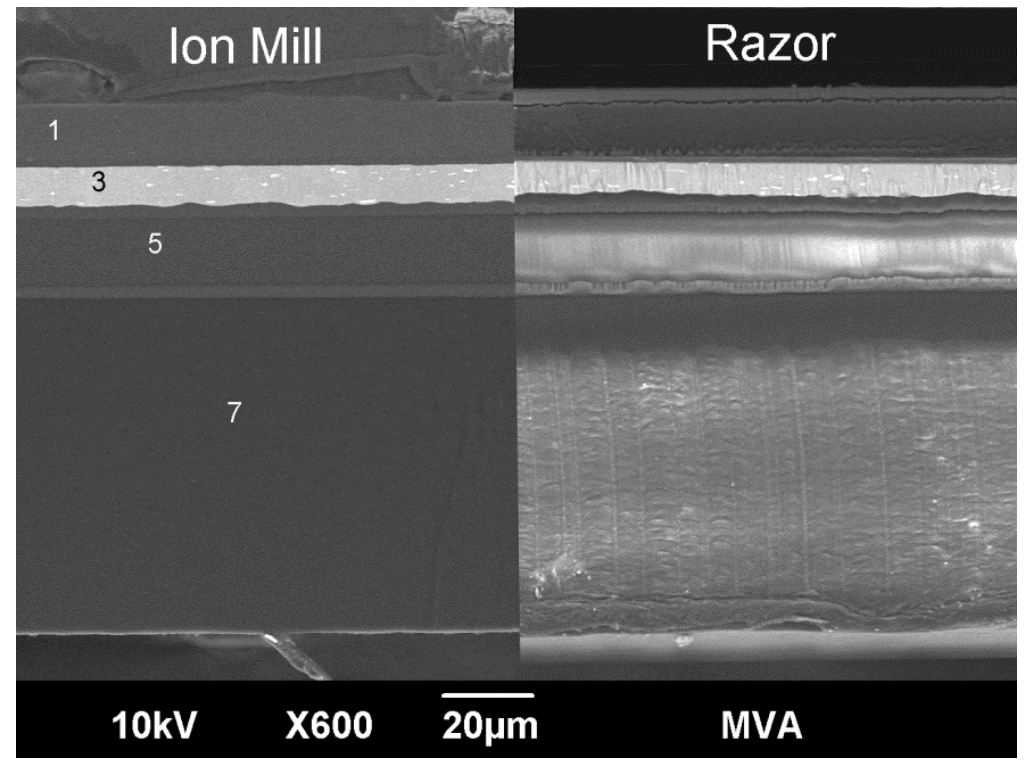

Figure 1. Backscattered electron image of multilayer foil laminate ion milled and razor cut cross sections. A combination of SEM-EDS, FTIR and CRM was used to characterize this material. Layers 2, 4 and 6 are less than 5 micrometers thick.

[1] http://www.fda.gov/medicaldevices/deviceregulationandguidance/overview/classifyyourdevice/ucm0 51512.htm

[2] Carlton, Robert Allan, "Pharmaceutical Microscopy", Springer, New York, 2011.

[3] Mathieu, C., "The Beam-Gas and Signal-Gas Interactions in the Variable Pressure Scanning Electron Microscope", Scanning Microscopy Vol. 13, No. 1, 1999 (Pages 23-41).

[4] Aldrich, D. Scott and Smith, Mark A. in , "Practical Guide to Infrared Microspectroscopy", Marcel Dekker,Inc. , New York, 1995, p323. 